07

\title{
Особенности электрон-ионного теплообмена в условиях интенсивного фотовозбуждения диэлектриков ультракороткими лазерными импульсами
}

\author{
() Д.С. Поляков ${ }^{1}$, Е.Б. Яковлев ${ }^{1}$, Д.С. Иванов ${ }^{2}$ \\ ${ }^{1}$ Санкт-Петербургский национальный исследовательский университет \\ информационных технологий, механики и оптики \\ 2 Университет Кассель, 34109, Кассель, Германия \\ E-mail: polyakovdmitry1988@gmail.com
}

Поступило в Редакцию 29 марта 2016 г.

\begin{abstract}
Проведен анализ скорости обмена энергией между перегретыми электронами и решеткой при воздействии ультракороткого лазерного импульса на диэлектрики для случая рассеяния электронов на деформационном потенциале акустических фононов и поляризационном потенциале оптических фононов. Определены температурные и концентрационные зависимости возникающего теплового потока, предложены аналитические выражения для их аппроксимации. Приведены оценки для диоксида кремния.
\end{abstract}

DOI: 10.21883/PJTF.2017.05.44359.16271

Поглощение ультракороткого лазерного импульса в полупроводниках и диэлектриках сопровождается интенсивной генерацией электронов и дырок, концентрация которых может достигать значений порядка $10^{21} \mathrm{~cm}^{-3}[1,2]$. При этом их температура в течение некоторого промежутка времени существенно превосходит температуру решетки. В таких условиях возникает поток энергии от горячих носителей к решетке $I_{e i}$, который традиционно представляется в виде произведения постоянного коэффициента $G$ на разность между электронной и решеточной температурами [3]. Однако, как показывают расчеты потока $I_{e i}$, проведенные в работах $[4,5]$ для некоторых металлов, коэффициент $G$ не является постоянным и зависит от электронной температуры. Для ряда важных с практической точки зрения полупроводников и диэлектриков детальные вычисления потока $I_{e i}$ отсутствуют, а в работах по модели- 
рованию воздействия ультракоротких импульсов на такие материалы используют феноменологические соотношения [6,7]. Таким образом, целью настоящей работы является получение теоретических оценок скорости электрон-ионного теплообмена и выявление ее температурной и концентрационной зависимостей на примере диоксида кремния.

Для энергии, передаваемой электронами проводимости в единицу времени единице объема решетки, справедливо выражение [5]

$$
\begin{aligned}
I_{e i}= & \sum_{j} \int \hbar \Omega_{j}(\mathbf{q}) w_{j}(\mathbf{q}) f(\mathbf{p})\left((1-f(\mathbf{p}-\mathbf{q}))\left(N_{j}(\mathbf{q})+1\right) \delta(E(\mathbf{p}-\mathbf{q})\right. \\
& \left.+\hbar \Omega_{j}(\mathbf{q})-E(\mathbf{p})\right)-(1-f(\mathbf{p}+\mathbf{q})) N_{j}(\mathbf{q}) \delta(E(\mathbf{p}+\mathbf{q}) \\
& \left.\left.-\hbar \Omega_{j}(\mathbf{q})-E(\mathbf{p})\right)\right) \frac{2 d \mathbf{p} d \mathbf{q}}{(2 \pi \hbar)^{6}}
\end{aligned}
$$

где $f(\mathbf{p})$ - функция распределения электронов, $N(\mathbf{q})$ - функция распределения фононов, $w(\mathbf{q})$ определяет вероятность поглощения (испускания) электроном фонона, $E(\mathbf{p})$ - энергия электрона, $\Omega(\mathbf{q})-$ частота фонона, $\mathbf{p}-$ импульс электрона, $\mathbf{q}-$ импульс фонона, $\hbar-$ приведенная постоянная Планка, индекс $j$ нумерует ветви фононного спектра, с которыми взаимодействуют электроны.

Для упрощения выражения (1) сделаем следующие допущения. Вопервых, функции распределения электронов и фононов будем полагать квазиравновесными:

$$
\begin{gathered}
f(E(\mathbf{p}))=\left(\exp \left(\frac{E(\mathbf{p})-\mu\left(n_{e}, T_{e}\right)}{k T_{e}}\right)+1\right)^{-1}, \\
N(\Omega(\mathbf{q}))=\left(\exp \left(\frac{\hbar \Omega(\mathbf{q})}{k T_{i}}\right)-1\right)^{-1},
\end{gathered}
$$

где $\mu-$ химический потенциал, $n_{e}-$ концентрация электронов, $T_{e}-$ электронная температура, $T_{i}$ - температура решетки, $k-$ постоянная Больцмана. Обоснование этого допущения для электронов можно найти в работах $[8,9]$. Во-вторых, дисперсионные зависимости для электронов и фононов примем в виде $E(\mathbf{p})=\left(2 m_{e}\right)^{-1} p^{2}, \Omega(\mathbf{q})=\hbar^{-1} s q$ для акустических фононов, $\Omega(\mathbf{q})=\Omega_{0}$ для оптических фононов, где $p=|\mathbf{p}|$,

Письма в ЖТФ, 2017, том 43, вып. 5 
$q=|\mathbf{q}|, m_{e}$ - эффективная масса электрона, $s-$ скорость звука, $\Omega_{0}$ - частота оптического фонона, считающаяся не зависящей от $q$. B-третьих, будем рассматривать рассеяние электронов на деформационном потенциале акустических фононов (DA) и поляризационном потенциале оптических фононов (PO). Оба этих механизма рассеяния имеют место в $\mathrm{SiO}_{2}$ (см., например, [10,11]). Согласно [12], вероятности $w(\mathbf{q})$ в этом случае будут иметь вид

$$
w_{D A}(\mathbf{q})=\frac{\pi D^{2}}{\hbar \rho s} q, \quad w_{P O}(\mathbf{q})=\hbar \Omega_{0} \frac{\pi e^{2}}{2 \varepsilon_{0}}\left(\frac{1}{\varepsilon_{\infty}}-\frac{1}{\varepsilon}\right) q^{-2},
$$

где $D-$ константа деформационного потенциала, $\rho-$ плотность, $e-$ заряд электрона, $\varepsilon_{0}$ - электрическая постоянная, $\varepsilon_{\infty}-$ высокочастотная диэлектрическая проницаемость, $\varepsilon-$ статическая диэлектрическая проницаемость.

С учетом сделанных допущений выражение (1) можно упростить. Для взаимодействия электронов с акустическими и оптическими фононами получаем

$$
\begin{gathered}
I_{e i}^{D A}=I_{e i}^{D A+}-I_{e i}^{D A-}, \\
I_{e i}^{D A \pm}=\frac{m_{e} D^{2}}{8 \pi^{3} \hbar^{7} \rho} \int_{0}^{\infty} f p d p \int_{0}^{q_{\max }^{ \pm}(p)}(1-f(E \mp s q))\left(N+\frac{1}{2} \pm \frac{1}{2}\right) q^{3} d q \\
I_{e i}^{P O}=I_{e i}^{P O^{+}}-I_{e i}^{P O^{-}}, \\
I_{e i}^{P O^{ \pm}}=\frac{m_{e} \Omega_{0}^{2} e^{2}\left(\varepsilon-\varepsilon_{\infty}\right)}{2(2 \pi \hbar)^{3} \varepsilon_{0} \varepsilon \varepsilon_{\infty}}\left(N\left(\Omega_{0}\right)+\frac{1}{2} \pm \frac{1}{2}\right) \\
\quad \times \int_{\left(\frac{1}{2} \pm \frac{1}{2}\right) \sqrt{2 m_{e} \hbar \Omega_{0}}}^{\infty} f\left(1-f\left(E \mp \hbar \Omega_{0}\right)\right) \ln \left(\frac{q_{\max }^{ \pm}(p)}{q_{\min }^{ \pm}(p)}\right) p d p
\end{gathered}
$$

Пределы интегрирования в выражении (2) задаются следующими выражениями:

$$
q_{\max }^{+}(p)= \begin{cases}2 p-2 m_{e} s, & 2 p \leqslant q_{D}+2 m_{e} s, \\ q_{D}, & 2 p>q_{D}+2 m_{e} s,\end{cases}
$$

3 Письма в ЖТФ, 2017, том 43, вып. 5 


$$
q_{\max }^{-}(p)= \begin{cases}2 p+2 m_{e} s, & 2 p \leqslant q_{D}+2 m_{e} s, \\ q_{D}, & 2 p>q_{D}+2 m_{e} s,\end{cases}
$$

а значения аргументов логарифмов в формуле (3) определяются по соотношениям

$$
\begin{gathered}
q_{\max }^{+}(p)= \begin{cases}p+\sqrt{p^{2}-2 m_{e} \hbar \Omega_{0}}, & 2 \sqrt{2 m_{e} \hbar \Omega_{0}}<2 p \leqslant q_{D}, \\
q_{D}, & 2 p>q_{D},\end{cases} \\
q_{\max }^{-}(p)= \begin{cases}p+\sqrt{p^{2}+2 m_{e} \hbar \Omega_{0}}, & 2 p \leqslant q_{D}, \\
q_{D}, & 2 p>q_{D},\end{cases} \\
q_{\min }^{+}(p)=p-\sqrt{p^{2}-2 m_{e} \hbar \Omega_{0}}, \quad q_{\min }^{-}(p)=-p+\sqrt{p^{2}+2 m_{e} \hbar \Omega_{0}},
\end{gathered}
$$

где $q_{D}-$ дебаевский импульс.

В случае высоких электронных температур, когда уместно пренебречь единицей в знаменателе $f(E)$, можно получить аналитические аппроксимации выражений (2) и (3):

$$
\begin{gathered}
\left.I_{e i}^{D A}\right|_{H T}=\frac{D^{2} m_{e}}{\rho} \frac{n_{e} q_{D}^{3}}{4 \pi \hbar^{4} \Gamma(1.5)} C^{-\frac{3}{2}}(1-(C+1) \exp (-C)), \\
\left.I_{e i}^{P O}\right|_{H T}=m_{e} \Omega_{0}^{2} \frac{n_{e} e^{2}}{2 \pi \varepsilon_{0} \Gamma(1.5)}\left(\frac{1}{\varepsilon_{\infty}}-\frac{1}{\varepsilon}\right) \frac{\sqrt{C}}{q_{D}}\left(\ln \left(\frac{q_{D}}{\alpha}\right)-\frac{\sqrt{C}}{2}\right),
\end{gathered}
$$

где $C=q_{D}^{2}\left(8 m_{e} k T_{e}\right)^{-1}, \alpha=\sqrt{2 m_{e} \hbar \Omega_{0}}, \Gamma(x)$ - гамма-функция.

Из (4) и (5) видно, что величина $I_{e i}$ растет линейно с ростом концентрации электронов в высокотемпературном приближении. Также существенно, что в выражениях (4) и (5) отсутствует решеточная температура. Зависимость от электронной температуры более сложная. Для ее иллюстрации на рис. 1 построены зависимости величины $I n=\left.I_{e i}\right|_{H T} n_{e}^{-1}$, вычисленные в соответствии с формулами (4) и (5) при высоких электронных температурах для следующего набора типичных значений параметров: $D=9 \mathrm{eV}, s=9000 \mathrm{~m} / \mathrm{s}, q_{D}=1.5 \cdot 10^{-24} \mathrm{~kg} \cdot \mathrm{m} / \mathrm{s}$, $\rho=2330 \mathrm{~kg} / \mathrm{m}^{3}, m_{e}=m_{0}, \varepsilon_{\infty}=2.9, \varepsilon=3.9, \hbar \Omega_{0}=100 \mathrm{meV}$. Из рис. 1 видно, что температурная зависимость величины $I n$ для случая рассеяния электронов на акустических фононах в рассмотренном диапазоне температур слабая. При больших $T_{e}$ справедливо разложение $\exp (-C) \approx 1-C$, соответственно, как следует из формулы (4),

Письма в ЖТФ, 2017, том 43, вып. 5 


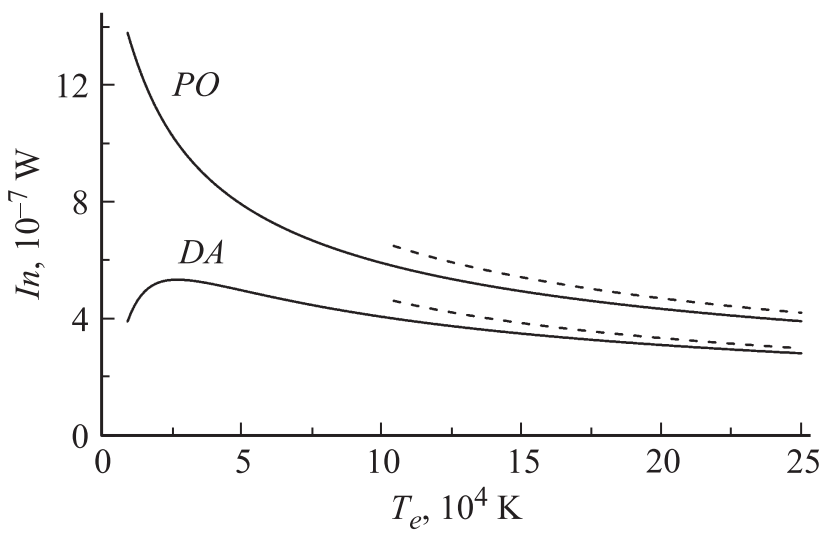

Рис. 1. Характерные температурные зависимости величины In для случая рассеяния на акустических фононах (деформационный потенциал, кривая $D A$ ) и оптических фононах (поляризационный потенциал, кривая $P O$ ), пунктиром показаны высокотемпературные асимптотики, имеющие обратную корневую зависимость $\left(\sim T_{e}^{-1 / 2}\right)$.

в высокотемпературном пределе $I n \sim T_{e}^{-1 / 2}$. В случае рассеяния на оптических фононах зависимость In от $T_{e}$ более резкая, однако в пределе высоких температур In $\sim T_{e}^{-1 / 2}$, так же как и для случая рассеяния на акустических фононах.

Приведем оценки скорости обмена энергией между электронами и решеткой для практически важного случая диоксида кремния. В $\mathrm{SiO}_{2}$ имеется взаимодействие как с акустическими, так и с оптическими фононами. Обычно рассматривают две ветви оптических фононов с энергиями 153 и $63 \mathrm{meV}$ и акустическую ветвь с $s=5935 \mathrm{~m} / \mathrm{s}$ и $q_{D}=2.6 \cdot 10^{-24} \mathrm{~kg} \cdot \mathrm{m} / \mathrm{s}$ [11]. Остальные параметры следующие: $D=6 \mathrm{eV}[11], \varepsilon_{\infty}=2.9, \varepsilon=3.9, \rho=2649 \mathrm{~kg} / \mathrm{m}^{3}, m_{e}=0.5 m_{0}[13]$.

Рис. 2 иллюстрирует вклад различных механизмов рассеяния электронов в скорость обмена энергией между перегретыми электронами и решеткой. Как видно, основной вклад в области низких электронных температур в величину $I_{e i}$ дает рассеяние на высокоэнергичной ветви оптических фононов. В области высоких $T_{e}$ становится существенным учитывать взаимодействие с акустическими фононами. На

$3^{*}$ Письма в ЖТФ, 2017, том 43, вып. 5 


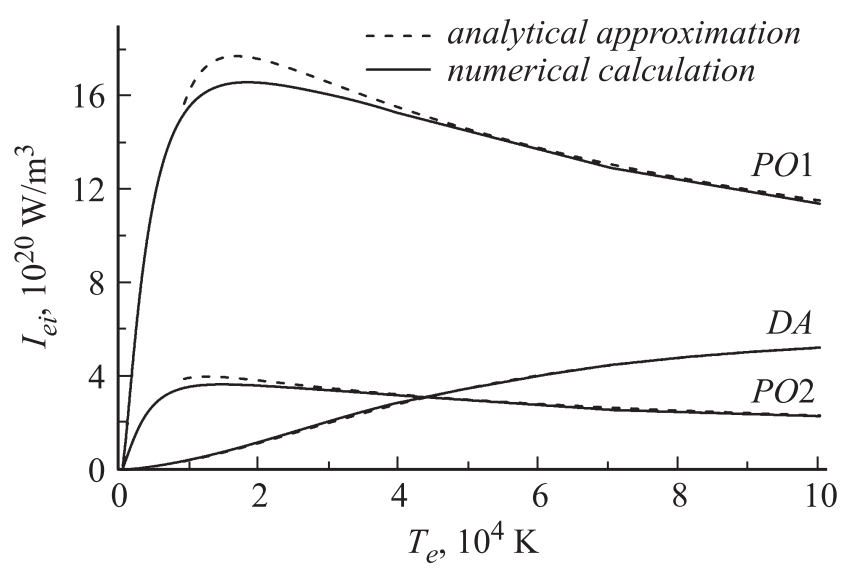

Рис. 2. Вклад различных механизмов рассеяния электронов на фононах в $\mathrm{SiO}_{2}$ в скорость теплообмена между электронами и решеткой для $n_{e}=10^{21} \mathrm{~cm}^{-3}$ : DA - вклад акустических фононов, PO1 - вклад оптических фононов с энергией $153 \mathrm{meV}$, PO2 - вклад оптических фононов с энергией $63 \mathrm{meV}$, сплошные кривые - численный расчет по формулам (2) и (3), пунктирные кривые - расчет по формулам (4) и (5).

рис. 3 показаны зависимости скорости электрон-ионного теплообмена для $\mathrm{SiO}_{2}$ от электронной температуры при различных концентрациях, рассчитанные по формулам (2), (3), и их сравнение с аналитическими аппроксимациями (4) и (5). Из рисунка видно, что для достигаемых в экспериментах по облучению $\mathrm{SiO}_{2}$ ультракороткими лазерными импульсами концентраций фотовозбужденных электронов порядка $10^{20}-10^{21} \mathrm{~cm}^{-3}$ [2] и электронных температур порядка $10^{4} \mathrm{~K}$ предложенные в работе аналитические аппроксимации (4) и (5) дают приемлемый результат. Конечно, реальная погрешность вычисления величины потока $I_{e i}$ может быть связана с нарушением допущений, сделанных при упрощении исходного выражения (1). Например, значение эффективной массы у высокоэнергичного электрона может отличаться от значения эффективной массы электрона у дна зоны проводимости. Соответственно представленные в работе расчеты для высоких электронных температур носят оценочный характер. Тем не менее, ввиду отсутствия надежных экспериментальных данных и более

Письма в ЖТФ, 2017, том 43, вып. 5 


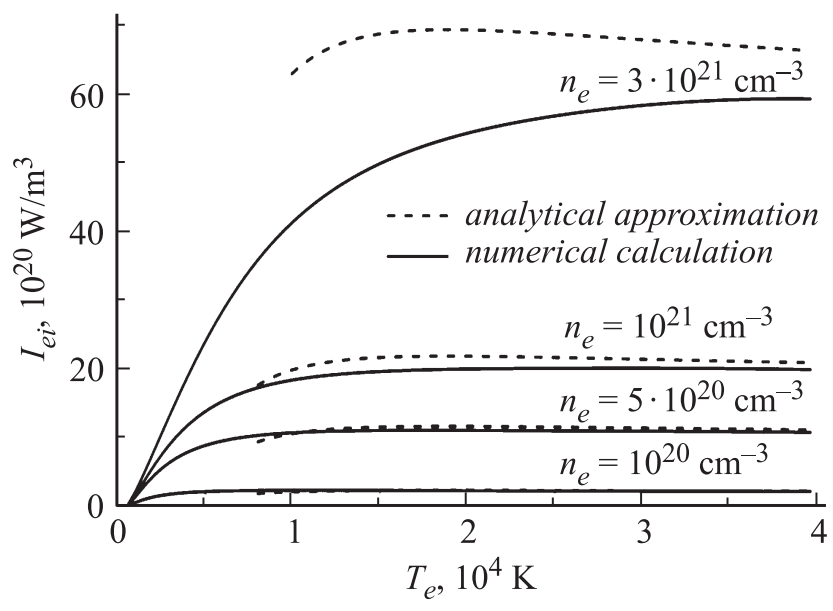

Рис. 3. Зависимости скорости электрон-ионного теплообмена в $\mathrm{SiO}_{2}$ от электронной температуры при различных концентрациях: сплошные кривые численный расчет по формулам (2) и (3), пунктирные кривые - расчет по формулам (4) и (5).

детальных вычислений величины потока $I_{e i}$, полагаем, что представленные в работе результаты будут полезны для количественного анализа воздействия сверхкоротких лазерных импульсов на диэлектрики (в частности, $\left.\mathrm{SiO}_{2}\right)$.

Таким образом, в работе получены температурные и концентрационные зависимости потока энергии от горячих электронов, сгенерированных при воздействии ультракороткого лазерного импульса на диэлектрики, к решетке для случаев рассеяния электронов на деформационном потенциале акустических фононов и поляризационном потенциале оптических фононов. Показано, что величина потока не является пропорциональной разности между электронной и решеточной температурами, и это следует учитывать при моделировании воздействия ультракоротких лазерных импульсов на диэлектрики.

Работа выполнена при поддержке гранта РНФ № 14-12-00351 и при государственной финансовой поддержке ведущих университетов РФ (субсидия 074-U01).

Письма в ЖТФ, 2017, том 43, вып. 5 


\section{Список литературы}

[1] Ионин А.А., Кудряшов С.И., Макаров С.В и др. // Письма в ЖЭТФ. 2012. T. 96. B. 6. C. 413-418.

[2] Mao S.S., Quere F., Guizard S. et al. // Appl. Phys. A. 2004. V. 79. P. 16951709.

[3] Вейко В.П., Либенсон М.Н., Червяков Г.Г., Яковлев Е.Б. Взаимодействие лазерного излучения с веществом. Силовая оптика. М.: Физматлит, 2008. $312 \mathrm{c}$.

[4] Lin Z., Zhigilei L. // Phys. Rev. B. 2008. V. 77. P. 075133.

[5] Петров Ю.В., Иногамов Н.А., Мигдал К.П. // Письма в ЖЭТФ. 2013. Т. 97. B. 1. C. $14-31$.

[6] Булгакова Н.М., Стоян Р., Розенфельд А. // Квантовая электроника. 2010. T. 40. B. 11. C. $966-985$.

[7] Ramer A., Osmani O., Rethfeld B. // J. Appl. Phys. 2014. V. 116. P. 053508.

[8] Яковлев Е.Б., Поляков Д.С. // Известия вузов. Приборостроение. 2015. Т. 58. B. 8. C. 664-669.

[9] Scheblanov N., Silaeva E., Itina T. // Appl. Surf. Sci. 2012. V. 258. P. 9417-9420.

[10] Fischetti M.V., DiMaria D.J., Brorson S.D. et al. // Phys. Rev. B. 1985. V. 31. P. 8124-8142.

[11] Kaiser A., Rethfeld B., Vicanek M., Simon G. // Phys. Rev. B. 2000. V. 61. P. 11437-11450.

[12] Басс Ф.Г., Гуревич Ю.Г. Горячие электроны и сильные электромагнитные волны в плазме полупроводников и газового разряда. М.: Физматлит, 1975. 399 c.

[13] Некрашевич С.С., Гриценко В.А. // ФТТ. 2014. Т. 56. В. 2. С. 209-223. 